\title{
Chronic Sclerosing Sialadenitis
}

National Cancer Institute

\section{Source}

National Cancer Institute. Chronic Sclerosing Sialadenitis. NCI Thesaurus. Code C82887.

A chronic fibrotic inflammatory process affecting the salivary gland. Signs and symptoms include firm and painful swelling of the salivary gland, often associated with the presence of salivary gland stones. 